\title{
Contagem, isolamento e identificação de Bacillus cereus em condimentos preparados, utilizados em embutido cárneo (mortadela)*
}

\author{
Counting, isolation and identification of Bacillus cereus in prepared \\ condiments, used in sausage (mortadella)
}

\author{
Marília dos Santos Pereira, ${ }^{\star \star}$ Luiz Antônio Trindade de Oliveira, ${ }^{* \star *}$ Robson Maia Franco, ${ }^{\star \star *}$ José Carlos \\ Albuquerque do Prado Carvalho***
}

\begin{abstract}
Resumo
Os condimentos vegetais têm sido utilizados pelo homem desde a mais remota antigüidade. São usados para modificar, de acordo com a vontade do homem, a apetecibilidade e a insipidez dos alimentos, bem como preservar alguns deles. Com o intuito de avaliar as condições higiênico-sanitárias dos condimentos preparados utilizados na elaboração de embutido cárneo (mortadela), analisoaram-se 38 amostras de compostos de sal, alho, noz-moscada, cravo, coentro, orégano, pimenta em pó e em pasta, procedentes de estabelecimento industrial submetido à Inspeção Federal, quanto a Contagem de Bacillus cereus viáveis em placas. Isso se justifica pela possibilidade dos mesmos participarem da contaminação do produto final, além de fornecer subsídios para uma reflexão no sentido da criação de padrão microbiológico referente ao Bacillus cereus, tendo em vista a sua importância no processo de toxinfecções alimentares. O número de Unidades Formadoras de Colônias por grama do produto (UFC/g) médio foi de 4,60 Log, sendo que $25 \%$ das amostras positivas apresentaram resultados iguais ou superiores a este. Das amostras positivas, em $76,6 \%$ confirmaram-se bioquimicamente a presença de Bacillus cereus. Os resultados denotam condições higiênico-sanitárias suspeitas das amostras, além de demonstrarem a factibilidade da presença de Bacillus cereus em condimentos preparados, significando risco à saúde do consumidor. Tomando como base a Portaria 451/97 do Ministério da Saúde, os valores obtidos em $25 \%$ das amostras positivas caracterizam o produto como "produto impróprio para o consumo" e "potencialmente capaz de causar toxinfecção alimentar".
\end{abstract}

Palavras-chave: contagem; isolamento; identificação; Bacillus cereus; condimentos; embutido.

\section{Introdução}

A utilização dos condimentos vegetais em alimentos desenvolveu-se de tal forma no século XX, que, a partir de 1953, passaram a ser empregados industrialmente, sendo definidos, segundo Guarino e Gray apud Vanderzant e Splittstoesser (1992), como temperos e misturas destes, que podem ser constituídos de outros acentuadores e potencializadores, com o objetivo de aumentar o sabor do alimento. Os mesmos definem tempero (especiaria) como plantas secas ou partes de plantas que, sozinhas ou misturadas, inteiras ou moídas, são usadas para temperar e conferir aroma ou cor a alimentos e bebidas.

$\mathrm{Na}$ indústria da carne os condimentos vegetais apresentam uso freqüente na elaboração de vários produtos, tais como os embutidos cárneos.

Segundo Jay, 1973, no caso de embutidos cárneos, grandes quantidades de microrganismos podem ser carreados para os mesmos através dos ingredientes a eles adicionados.
A contaminação microbiana nos condimentos vegetais ou especiarias pode ocorrer pelo fato de serem produzidos em áreas onde as práticas são consideradas precárias e muitos são obtidos em locais onde o crescimento microbiano é favorecido (áreas quentes e úmidas), sendo as bactérias durante o processo de colheita e manuseio, facilmente distribuídas pelos mesmos (Guarino e Peppler apud Speck, 1976; Baxter e Holzapfel, 1982).

Segundo Frazier (1972), Bacillus cereus apresenta-se como um microrganismo telúrico, habitando o solo, vegetação e a água. Harmon e Goepfert apud Vanderzant e Splittstoesser (1992), consideram que este microrganismo se encontra amplamente distribuído na natureza e pode ser isolado, prontamente, de uma ampla variedade de alimentos.

Conforme Delazari et al. (1978), desde o início do presente século esta bactéria vem sendo incriminada em casos de surtos de toxinfecção alimentar. Os mesmos autores descrevem a facilidade com que os alimentos são contamina-

\footnotetext{
*Apoio financeiro do CNPq.

**Aluna de Pós-Graduação na Faculdade de Veterinária - Universidade Federal Fluminense.

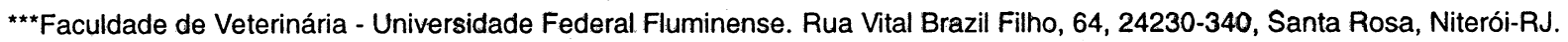


dos devido à característica telúrica do microrganismo. Gilbert e Parry (1977) explicam que valores baixos, menores que 2 Log10 Unidades Formadoras de Colônias (UFCs)/ g são, provavelmente, pouco significativos, mas, com grandes quantidades, especialmente maiores que 5 Log10 UFCs/g, há um risco significativo de que ocorra toxinfecção alimentar. Bryan (1980) analisa que são poucos os surtos confirmados envolvendo este microrganismo como agente causal. O autor considera que isto é, sem dúvida, decorrência da falha de investigadores e laboratórios. Mas Beckers (1982) cita que cerca de $30 \%$ de 42 incidentes envolvendo carne e derivados tiveram como agente etiológico - Bacillus cereus, e Shinagawa et al. (1988) concluíram que os produtos cárneos são contaminados com esporos de Bacillus cereus os quais alcançam o produto final processado, através dos aditivos a eles adicionados.

Segundo alguns autores, dentre eles Johnson, Nelson e Busta (1983), o Bacillus cereus é capaz de determinar dois tipos distintos de toxinfecção alimentar: uma Síndrome Tipo Diarréia (forma clássica) e Síndrome Tipo Emética.

Objetivamos avaliar as condições higiênico-sanitárias dos condimentos preparados utilizados na elaboração de embutido cárneo e a possibilidade dos mesmos participarem da contaminação do produto final, além de fornecer subsídios para uma reflexão, no que diz respeito à criação de padrões microbiológicos.

\section{Materiais e métodos}

O trabalho foi realizado no período de setembro a dezembro de 1992, no Laboratório de Controle Microbiológico de Produtos de Origem Animal do Departamento de Tecnologia dos Alimentos da Faculdade de Veterinária da Universidade Federal Fluminense, Niterói, RJ.

Analisaram-se 38 amostras de condimentos preparados (mistura de sal e condimentos vegetais), colhidas em um estabelecimento industrial submetido à Inspeção Federal e que os utiliza na elaboração de embutido cárneo (mortadela). Esses condimentos eram procedentes de um outro estabelecimento industrial, submetido, também, à Inspeção Federal, e que elabora e/ou manipula produtos de origem animal.

As amostras da mistura de condimentos composta de sal, alho, noz-moscada, cravo, coentro, orégano, pimenta em pó e em pasta foram transportadas para o laboratório nas condições originais em que são manipuladas, ou seja, em embalagens plásticas, hermeticamente fechadas e pesando aproximadamente 250 gramas, sendo identificadas e, posteriormente, analisadas.

Previamente às análises, assepticamente, amostras foram preparadas pesando-se uma alíquota de 25 gramas, os quais foram homogeneizadas juntamente com $225 \mathrm{~mL}$ de solução salina peptonada a $0,1 \%$ em homogeneizador "Waring blender" à velocidade máxima por 2 minutos seguido de 5 minutos de repouso para sedimentação de partículas mais grosseiras (Oliveira, 1983). A partir do sobrenadante obtido (diluição $10^{-1}$ ) procedeu-se a diluições até o nível de $10^{-6}$. A partir destas diluições procedeu-se a contagem, isolamento e identificação de Bacillus cereus conforme Refai (1979) e Brasil (1981), utilizando o Agar Polimixina Vermelho de Fenol Gema de Ovo - APVF.

\section{Resultados}

Nas 38 amostras de condimentos preparados analisadas, observou-se a presença de UFCs típicas isoladas em 21 amostras $(55,26 \%)$. Destas, 16 amostras $(76,19 \%)$ apresentaram UFCs, cujo perfil bioquímico observado sugere a identificação positiva de Bacillus cereus.

No Quadro 1 e com o auxílio do Gráfico 1, observa-se que os resultados obtidos na contagem desta bactéria apresentaram variação na faixa entre 1 Log10 à 6.08 Log10 UFCs/g, com uma média de 2,68 Log10 UFCs/g.

Quadro 1: Freqüência dos resultados das contagens de Bacillus cereus viáveis por intervalos de valores de Log 10

\begin{tabular}{cc}
\hline Freqüência das amostras / \% & Intervalo de Log 10 UFCs/g \\
\hline $22 / 57,89 \%$ & $0,00-0,99$ \\
$06 / 15,79 \%$ & $1,00-1,99$ \\
$05 / 13,16 \%$ & $2,00-2,99$ \\
$01 / 2,63 \%$ & $3,00-3,99$ \\
$0 / 0 \%$ & $4,00-4,99$ \\
$03 / 7,89 \%$ & $5,00-5,99$ \\
$01 / 2,63 \%$ & $6,00-6,99$ \\
\hline
\end{tabular}

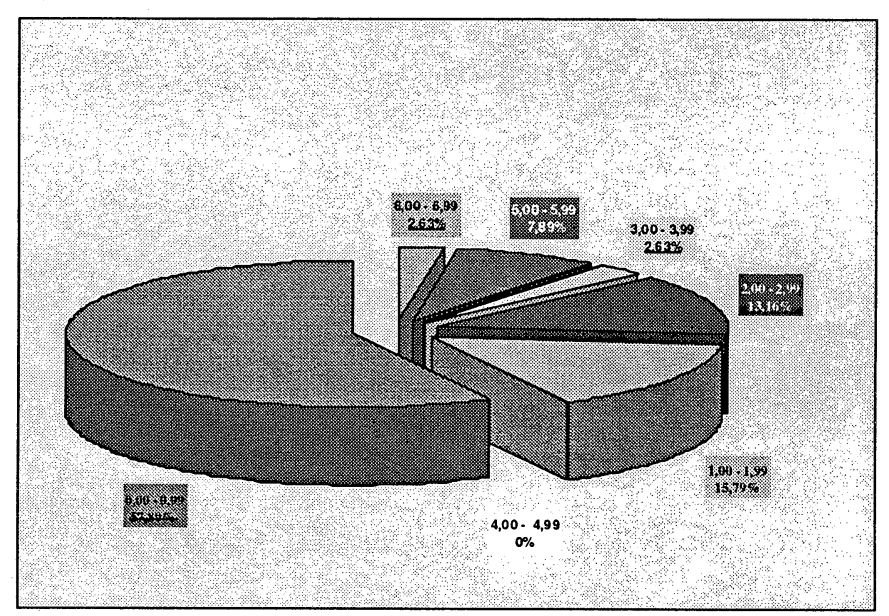

Gráfico 1: Freqüência dos resultados das contagens de Bacillus cereus viáveis por intervalos de valores de Log 10

\section{Discussão}

Em decorrência de sua constituição, os alimentos de origem animal são susceptíveis à deterioração (Shelf Life of Foods, 1981), e,vários são os agentes capazes de in- 
tervir em sua qualidade (Rozier, Carrier e Bolnot, 1982). Assim sendo, podemos suspeitar que tudo o que participe da elaboração do alimento e entre em contato com o mesmo, possa funcionar como fonte de contaminação, como é o caso dos aditivos usados na composição de embutidos cárneos, tais como os condimentos vegetais. Considera-se a potencialidade de tais aditivos veicularem microrganismos para os alimentos, pelo fato de sua maioria ser obtida em áreas tropicais e precárias, onde o crescimento bacteriano é favorecido e, desta forma, distribuído ao mesmo durante a colheita e manuseio (Oliveira, 1983; Baxter e Holzapfel, 1982).

No presente trabalho ficou comprovado, através da contagem, isolamento e identificação, a presença de Bacillus cereus em condimentos preparados, utilizados na elaboração de embutido cárneo (mortadela). Os resultados obtidos nas amostras analisadas variaram de 1,00 Log10 a 6,08 Log10 UFCs/g (Quadro1 e Gráfico 1), tendo sido encontrada um média de 2,68 Log10 UFCs/g, enquanto Komuna et al., 1988, analisando amostras de aditivos, obtiveram resultados dentro da faixa observada por nós, estando estes variando de 2,00 Log10 a 4,00 Log10UFCs/ $\mathrm{g}$, sendo os valores mais elevados $(4,00 \mathrm{Log} 10 \mathrm{UFCs} / \mathrm{g})$ encontrados entre os condimentos. Tendo como base 0 ICMSF, 1978, observa-se que os resultados obtidos neste trabalho são superiores aos encontrados quanto à contaminação dos alimentos pelos aditivos a eles adicionados, e que um percentual significativo destes resultados
(25\%) indicam a possibilidade de se determinar uma toxinfecção no ingestor.

\section{Conclusões}

Os resultados obtidos no presente trabalho permitem concluir que:

a) as condições higiênico-sanitárias das amostras analisadas sugerem contaminação microbiológica durante a obtenção e/ou manuseio, bem como tratamento de limpeza insuficiente;

b) o isolamento de Bacillus cereus é factível em condimentos preparados, significando risco à saúde do consumidor;

c) $25 \%$ das amostras confirmadamente positivas apresentaram resultados iguais ou superiores a 4 Log10UFCs/ g, enquadrando-se assim, como "produto impróprio para o consumo" ou "potencialmente capaz de causar enfermidades transmitidas por alimentos", segundo a Portaria 451/97 do Ministério da Saúde (Brasil, 1997), no seu anexo III, item III; e

d) é importante salientar a necessidade da continuidade de análises com vistas a obter cada vez mais dados sobre a presença de Bacillus cereus e capazes de induzir a uma análise sistemática em condimentos preparados utilizados na indústria, bem como em condimentos encontrados no comércio, visando à criação de padrões microbiológicos nacionais.

\section{Abstract}

The vegetable condiments have been used by man since old times. It is used to transform, depending on man intention, the tastefulness of foods and preserve some of them. With intention of estimate hygienic-sanitary conditions of prepared condiments useds in that product working out and the possibility of them help the product contamination, besides give conditions to think about microbiological standards creation to Bacillus cereus (considering its importance in foods toxinfections), were analyzed 38 samples of prepared condiment composite with salt, garlic, nutmeg, clove, coriander, powdered and paste pepper. The samples were applied to make stuffed meat products (long Italian sausage) and they were collected in industries under Federal Inspection and the analyses consisted of viable Bacillus cereus count. The number of middle Count Forming Units per gram (CFU/g) was 4,60 log10. Twenty five percent of positive samples had results similars or biggers than that. In the positive samples, $76,6 \%$ were confirmed Bacillus cereus presence. The results show us that the hygienic-sanitary conditions of the samples were suspects, besides to demonstrate the possibility of Bacillus cereus presence in prepareds condiments meaning public health hards. Considering the government order 451/97 of Health Ministry, $25 \%$ of samples results characterize the product as improper to consume and potentially capable produce food toxinfections.

Keywords: count; Isolament; Identification; Bacillus cereus; condiments; stuffed meat.

\section{Referências bibliográficas}

BAXTER,R. e HOLZAPFEL, W.H. A microbial investigation of selected spices, herbs and additives in South Africa. Journal of Food Science, v. 47, n. 2, p. 570-574, 578, Feb. 1982.

BECKERS, H.J. Incidence of foodborne disease in the Netherlands: Annual Summary 1979. Journal of Food Protection, v. 45, n. 14, p. 1338-1341, Dec. 1982.
BRASIL. Secretaria Nacional de Defesa Agropecuária. Métodos Analíticos Oficiais para Controle de Produtos de Origem Animal e seus Ingredientes: Métodos Microbiológicos, Brasília, DF : Ministério da Agricultura, 1981. 2 v., v. 1.

BRASIL. Secretaria de Vigilância Sanitária. Portaria 451 de 19 de setembro 1997. Princípios Gerais para o Estabelecimento de Critérios e Padrões Microbiológicos para Alimentos. Diário Oficial da União. Brasília, DF. p. 21005-21012, 22 set. 1997. 
BRYAN, F.L. Foodborne diseases in the United States associated with meat and poultry. Journal of Food Protection, v. 43 , n. 2 , p. $140-150$, Feb. 1980.

DELAZARI, I. et al. Bacillus cereus em alimentos desidratados. Boletim do Instituto de Tecnologia de Alimentos, Campinas, n. 60, p. 31-40, nov./dez. 1978.

FRAZIER, W.C. Microbiologia de los Alimentos. 2. ed. Zaragoza : Acribia, 1972. 512 p.

GILBERT, R.J. e PARRY, M.J. Serotypes of Bacillus cereus from outbreaks of food poisoning and from routine foods. J. Hygiene, Cambridge, n. 78, p. 69-78, 1977.

GUARINO, P.A. e GRAY, R.J.H. Spices and gums. In: VANDERZANT, C e SPLITTSTOESSER, D.F. Compendium of Methods for the Microbiological Examination of Foods. 3. ed. Washington: APHA, 1992. 1219 p. Cap. 52, p. 961-974.

GUARINO, P.A. e PEPPLER, H.J. Spices and condiments. In: SPECK, M.L. Compendium of Methods for the Microbiological Examination of Foods. Washington : APHA, 1976. Cap. 46, p. 568-573.

HARMON, S.M., GOEPFERT, J.M. e BENNETT, R.W. Bacillus cereus. In: VANDERZANT, C e SPLITTSTOESSER, D.F. Compendium of Methods for the Microbiological Examination of Foods. 3. ed. Washington: APHA, 1992. 1219 p. Cap. 35, p. 593-604.

INTERNATIONAL Commission on the Microbiological Specification for Foods. Microrganisms in Foods: Their - significance and methods of enumeration. Zaragoza : Acribia, 1978.
INTERNATIONAL Commission on the Microbiological Specification for Foods. Ecología Microbiana de los Alimentos: Productos alimenticios. Zaragoza : Acribia, 1985. 989 p.

JAY, J.M. Microbiología Moderna de los Alimentos. 2. ed. Zaragoza : Acribia, 1973. 319 p.

JOHNSON, K.M., NELSON, C.L. e BUSTA, F.F. Influence of temperature on germination and growth of spores of emetic and diarrheal strains of Bacillus cereus in a broth medium and in rice. J. Food Science, Minnesota, v. 48, n. 1, p. 286287, jan. 1983.

KOMUNA, $\mathrm{H}$. et al. Ocorrence of Bacillus cereus in meat products, raw meat and meat product additives. Journal of Food Protection, v. 51, n. 4. p. 324-326, abr. 1988.

OLIVEIRA, L.A.T. Anaeróbios em especiarias utilizadas em embutidos cárneos. Niterói, 1983. 66 p. Tese (Doutorado em Ciências - Microbiologia) - Universidade Federal do Rio de Janeiro.

REFAI, M.K. Manuals of food quality control. 4. Microbiological analysis. Roma: Food and Agriculture Organization of the United Nations. 1979. 76 p. (FAO Food and Nutrition Paper, 14/4).

ROZIER, J., CARRIER, V. e BOLNOT, F. Dégradation de la qualité des aliments par les microrganismes. RTVA, v. 21, n. 180, p. 3-13, jul./ago. 1982.

"SHELF-LIFE" of food. Food Technology in the New Zeland, n. 2, p. 5-11, fev. 1981.

SHINAGAWA, K. et al. Enumeration of aerobic spore-formers and Bacillus cereus in meat product additives. Journal of Food Protection, Ames, v. 51, n. 8, p. 648-650, ago. 1988.

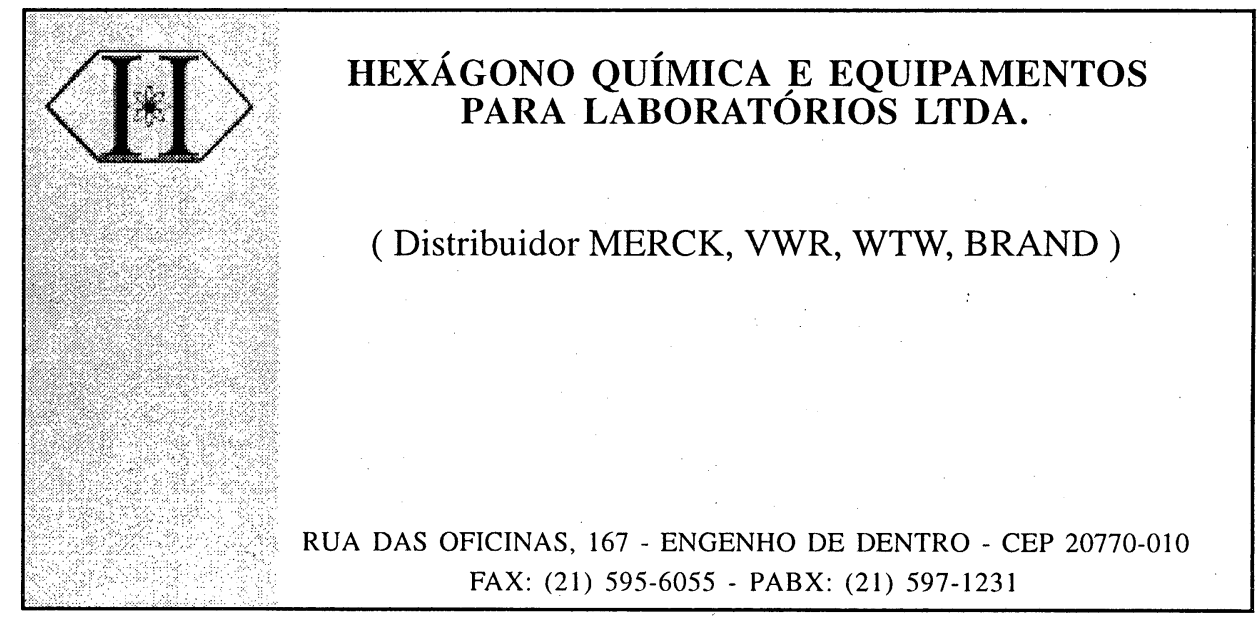

